\title{
Retinol-binding protein 4 is closely correlated to blood pressure level and E/A in untreated essential hypertension patients
}

\author{
Xiuzhen Li ${ }^{1,2 \#}$, Shushu Zhu ${ }^{1,2 \#, ~ G u o x i n ~ S o n g}{ }^{3}$, Kangzhen Zhang ${ }^{2}$, Wei Gao ${ }^{4}$, Jingjing Huang ${ }^{2,5}$, \\ Xiang $\mathbf{L u}^{6,7}$
}

${ }^{1}$ Department of Cardiology, The Second Affiliated Hospital of Nanjing Medical University, Nanjing 210011, China; ${ }^{2}$ The Second Clinical Medical College of Nanjing Medical University, Nanjing 210011, China; ${ }^{3}$ Department of Pathology, The First Affiliated Hospital with Nanjing Medical University, Nanjing 210029, China; ${ }^{4}$ Department of Geriatrics, Sir Run Run Hospital, Nanjing Medical University, Nanjing 211166 , China; ${ }^{5}$ Department of Geriatrics, The Second Affiliated Hospital of Nanjing Medical University, Nanjing 210011, China; ${ }^{6}$ Nanjing Medical University, Nanjing 210029, China; ${ }^{7}$ Key Laboratory for Aging and Disease, Nanjing Medical University, Nanjing 211166, China

Contributions: (I) Conception and design: X Lu, J Huang; (II) Administrative support: X Lu; (III) Provision of study materials or patients: X Li, S Zhu; (IV) Collection and assembly of data: X Li, K Zhang, G Song; (V) Data analysis and interpretation: X Li, K Zhang, W Gao; (VI) Manuscript writing: All authors; (VII) Final approval of manuscript: All authors.

\#These authors contributed equally to this work.

Correspondence to: Xiang Lu. Key Laboratory for Aging and Disease, Nanjing Medical University, Nanjing 211166, China. Email: luxiang66@njmu.edu.cn; Jingjing Huang. Department of Geriatrics, The Second Affiliated Hospital of Nanjing Medical University, The Second Clinical Medical College of Nanjing Medical University, Nanjing 210011, China. Email: jingjinghuang@njmu.edu.cn.

Background: Hypertension, a common chronic disease, is a leading cause of death and other cardiovascular
diseases. Recent studies show that an inflammatory factor named retinol binding protein 4 (RBP4) was
increased with cardiovascular diseases. However, the relationship between RBP4 and hypertension in patients
remains unclear.
Methods: The study cohort was composed of patients with essential hypertension (EH) and healthy control
(HC) subjects from the Second Affiliated Hospital of Nanjing Medical University [2017-2019]. The levels of
RBP4 and echocardiography were compared in the current study. Statistical differences between two groups
were analyzed using unpaired Student's $t$-tests and the correlation between the two variables adopts Pearson
correlation analysis. SPSS 23.0 was used for all statistical analysis. Results: Analysis of patient plasma samples revealed that RBP4 in EH group was greater than HC group $(\mathrm{P}<0.05)$. The area under the ROC curve was 0.717 . Specificity and sensitivity were $80.4 \%$ and $60.8 \%$, respectively. RBP4 had positive correlation with left ventricular systolic diameter (LVDs), interventricular septal thickness (IVS) and left ventricular posterior wall thickness (LVPW), negative correlation with left ventricular shortening fraction (FS) and ejection fraction $(\mathrm{EF})(\mathrm{P}<0.05)$, and no correlation with left ventricular end-diastolic diameter (LVDd) ( $>0.05)$. RBP4 was closely related with E/A, evaluation method of left ventricular diastolic function, in patients with EH.

Conclusions: RBP4 levels are closely correlated with blood pressure (BP) levels and might be involved in the regulation of left ventricular diastolic function in patients with $\mathrm{EH}$.

Keywords: Retinol binding protein 4 (RBP4); hypertension; left ventricular diastolic function

Submitted Sep 25, 2019. Accepted for publication Nov 05, 2019.

doi: 10.21037/apm.2019.11.07

View this article at: http://dx.doi.org/10.21037/apm.2019.11.07 


\section{Introduction}

Essential hypertension (EH) is a significant worldwide public health problem. The global prevalence of hypertension was estimated to be 1.13 billion in 2015 affecting more than 27 million of the population from the 2017 national census in china (1), high blood pressure (BP) is the most important risk factor for heart failure; and approximately $75 \%$ of heart failure cases have antecedent hypertension (2). The spontaneously hypertensive rat showed hypertrophy of heart for compensation, and demonstrated fibrosis and death of cardiac myocytes, and ultimately progressed to heart failure as shown by magnetic resonance imaging (3). Although heart failure on left ventricular systolic dysfunction has received more attention, diastolic dysfunction was not. In order to improve the prognosis of patients with $\mathrm{EH}$, it's important to identify the risk factors that are associated with diastolic dysfunction.

Retinol binding protein 4 (RBP4) is an adipokines and inflammatory factor secreted mainly by hepatocytes and adipocytes. It is closely related with insulin resistance, diabetes, coronary heart disease, heart failure and hypertension development (4-11). However, the relationship between RBP4 level and hypertension or the left ventricular diastolic function in patients with $\mathrm{EH}$ has rarely been reported. The aim of the study is to investigate the association between the levels of RBP4 and the BP levels or the left ventricular diastolic function in patients with $\mathrm{EH}$.

\section{Methods}

\section{Study population}

The data were collected from random participants in the Department of Cardiology, Second Affiliated Hospital of Nanjing Medical University from March 2017 to March 2019. Hypertensive patients without oral antihypertensive drugs $(n=255)$ were obtained from hypertensive patients. According to the consideration of medical ethics and medical style in our hospital, 74 patients were included (EH group). Following exclusion criteria: (I) secondary hypertension; (II) myocardiopathy, heart failure, kidney disease, stroke and other organ damage; (III) coronary artery disease ( $\geq 50 \%$ ); (IV) fasting blood glucose (FBG) $>6.1 \mathrm{mmol} / \mathrm{L}$ or thyroid gland disease; (V) tumor; (VI) infectious disease; (VII) pregnant or lactating. In comparison, the healthy control (HC) group recruited 46 healthy volunteers (HC group). The informed consent of patients was obtained before sample collection. Fasting blood samples were collected and centrifuged $(2,000 \mathrm{r} / \mathrm{min})$ for $10 \mathrm{~min}$ at $-4{ }^{\circ} \mathrm{C}$. The serum was collected into Eppendorf tubes and stored at $-80{ }^{\circ} \mathrm{C}$ pending to analysis. The current and previous medical histories, as well as the body weight and height were collected. FBG levels were measured using the glucose oxidase method. Serum total cholesterol (TC), triglyceride (TG), low density lipoprotein cholesterol (LDL-C) and high density lipoprotein cholesterol (HDL-C) contents were determined enzymatically using a kit (Beijing BHKT Clinical Reagent Co., Ltd, Beijing, China). The results were analyzed by an automated analyzer. Cardiac ultrasound results were obtained in nearly 3 months. Arterial BP was measured by taking the average value from at least two $\mathrm{BP}$ measurements in the sitting position during a 1-2 min interval.

\section{Determine serum RBP4 levels by ELISA kit}

All reagents, standard dilutions, and samples were brought to room temperature and prepared as directed in the product manual of RBP4 ELISA kit (Phoenix, USA).

\section{Echocardiography}

Echocardiography is a convenient, simple, noninvasive, accurate and inexpensive method to evaluate left ventricular diastolic function, which is widely used in clinical practice. In this study, the left ventricular diastolic function was evaluated by the echocardiography and represented as ratio of mitral valve early diastolic peak blood flow velocity (E) to mitral valve late diastolic peak blood flow velocity (A). Various echocardiographic indexes were measured on patients taking the left lateral decubitus position using color Doppler ultrasound diagnostic instrument (PHILIP Company) on multiple standard sections. Echocardiographic indexes acquired in this study include left ventricular end diastolic inner diameter (LVDd), left ventricular enddiastolic diameter (LVDs), interventricular septum thickness (IVS), left ventricular posterior wall thickness (LVPW), fractional shortening (FS), ejection fraction (EF). The most widespread paradigm for the assessment of ventricularvascular coupling is the ventricular (E)-arterial (A) elastance framework, which links mechanical performance of the ventricle to its oxygen consumption. E means the endsystolic elastance (slope of the end-systolic pressure-volume relation), a measure of ventricular contractility. A stands for arterial elastance (ratio of end-systolic pressure and stroke volume). 
Table 1 Clinical parameters of the study population (mean \pm standard deviation)

\begin{tabular}{|c|c|c|c|}
\hline Parameter & HC group $(n=46)$ & EH group $(n=74)$ & $P$ value \\
\hline Age (year) & $49.67 \pm 10.95$ & $49.85 \pm 11.43$ & 0.93 \\
\hline BMI $\left(\mathrm{kg} / \mathrm{m}^{2}\right)$ & $20.21 \pm 3.16$ & $24.96 \pm 3.35^{\star \star *}$ & 0.00 \\
\hline FBG (mmol/L) & $4.88 \pm 0.47$ & $5.14 \pm 0.58^{* *}$ & 0.01 \\
\hline $\mathrm{TC}(\mathrm{mmol} / \mathrm{L})$ & $4.76 \pm 0.84$ & $4.91 \pm 1.03$ & 0.42 \\
\hline $\mathrm{TG}(\mathrm{mmol} / \mathrm{L})$ & $1.20 \pm 0.51$ & $2.22 \pm 2.41^{\star \star *}$ & 0.00 \\
\hline $\mathrm{HDL}(\mathrm{mmol} / \mathrm{L})$ & $1.59 \pm 0.69$ & $1.21 \pm 0.41^{\star \star \star}$ & 0.00 \\
\hline LDL (mmol/L) & $2.78 \pm 0.54$ & $3.00 \pm 0.87$ & 0.22 \\
\hline $\mathrm{Cr}(\mu \mathrm{mol} / \mathrm{L})$ & $71.99 \pm 16.46$ & $77.68 \pm 17.92$ & 0.09 \\
\hline BUN (mmol/L) & $4.71 \pm 1.12$ & $5.24 \pm 1.39$ & 0.04 \\
\hline $\mathrm{EF}(\%)$ & $65.33 \pm 5.37$ & $64.62 \pm 5.11$ & 0.628 \\
\hline FS (\%) & $35.73 \pm 4.18$ & $35.33 \pm 3.98$ & 0.723 \\
\hline $\mathrm{SBP}(\mathrm{mmHg})$ & $114.22 \pm 10.64$ & $154.62 \pm 20.71^{\star \star \star}$ & 0.00 \\
\hline $\mathrm{DDBP}(\mathrm{mmHg})$ & $69.50 \pm 8.50$ & $94.95 \pm 10.61^{\star \star \star}$ & 0.00 \\
\hline MAP (mmHg) & $84.41 \pm 8.59$ & $114.84 \pm 11.94^{\star \star \star}$ & 0.00 \\
\hline
\end{tabular}

**, $\mathrm{P}<0.01 ;{ }^{* * *}, \mathrm{P}<0.001$. HC, healthy control; $\mathrm{EH}$, essential hypertension; BMI, body mass index; FBG, fasting blood glucose; TC, total cholesterol; TG, triglycerides; HDL-C, highdensity lipoprotein C; LDL-C, low-density lipoprotein C; $\mathrm{Cr}$, creatinine; EF, ejection fraction; FS, fractional shortening; SBP, systolic blood pressure; DBP, diastolic blood pressure; MAP, mean arterial pressure.

Table 2 The plasma levels of RBP4 in HC and EH groups (mean \pm standard deviation)

\begin{tabular}{lccc}
\hline Variable & HC group & EH group & P value \\
\hline RBP4 (mg/L) & $30.25 \pm 17.76$ & $38.40 \pm 15.76^{\star}$ & 0.01 \\
\hline
\end{tabular}

Analysis of the plasma of patients revealed that the levels of RBP4 in EH group were significantly $(P<0.05)$ higher than that in $\mathrm{HC}$ group ( $\left.{ }^{*}, \mathrm{P}<0.05\right)$. RBP4, retinol binding protein 4; $\mathrm{HC}$, healthy control; $\mathrm{EH}$, essential hypertension.

\section{Statistical analysis}

Continuous variables were summarized as mean $\pm \mathrm{SD}$ or as median values with interquartile ranges. Statistical differences were analyzed using unpaired Student's $t$-tests for two groups and the correlation between the two variables adopts Pearson correlation analysis. A $\mathrm{P}$ value $<0.05$ ( $^{*}, \mathrm{P}<0.05$; **, $\mathrm{P}<0.01$; ***, $\left.\mathrm{P}<0.001\right)$ indicates that there is significant statistical differences between two groups. All statistical analyses were performed using PASW

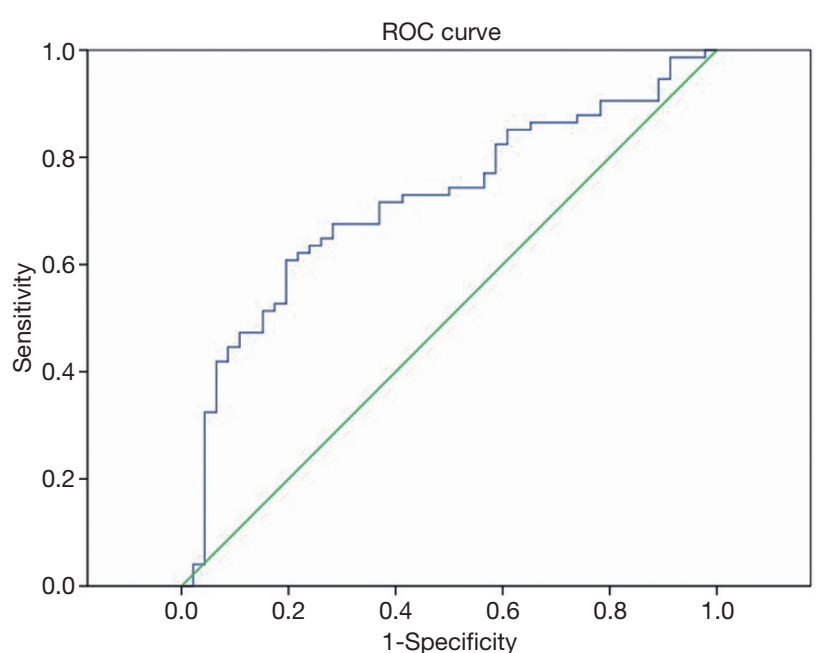

Figure 1 Receiver operating characteristic curves for the diagnostic accuracy of RBP4 for EH. Receiver operating characteristic curve demonstrated sensitivities and specificities of the RBP4 criteria in $\mathrm{EH}$. The area under the ROC curve is $0.717(\mathrm{P}<0.05)$. Specificity and sensitivity are $80.4 \%$ and $60.8 \%$, respectively. RBP4, retinol binding protein 4; EH, essential hypertension.

23.0 (IBM SPSS, Inc., Chicago, USA).

\section{Results}

In the clinical parameters of the study population, there is no significant difference of age, TC, LDL-C, EF, FS and creatinine $(\mathrm{Cr})$ between $\mathrm{EH}$ group and $\mathrm{HC}$ group $(\mathrm{P}>0.05)$; however, there were significant differences $(\mathrm{P}<0.05)$ of body mass index (BMI), FBG, blood urea nitrogen (BUN), TG, HDL-C, SBP, DBP and mean arterial pressure (MAP) between these two groups (Table 1). Analysis of the plasma of patients revealed that the serum RBP4 levels in $\mathrm{EH}$ group were significantly $(\mathrm{P}<0.05)$ higher than that in $\mathrm{HC}$ group (Table 2). We then analyzed the diagnostic accuracy of RBP4 for EH. As shown in Figure 1, ROC curve analysis showed that the optimal cut-off value of RBP4 for the prediction of EH was $33.71 \mathrm{mg} / \mathrm{L}$, with a sensitivity of $80.4 \%$ and a specificity of $60.8 \%$ (area under the curve $=0.717,95 \%$ CI: $0.62-0.81, \mathrm{P}<0.05$ ). We next evaluated the association of RBP4 with cardiac structure parameters in EH patients (Figure 2). In EH group, the levels of RBP4 were no correlation with LVDd ( $\mathrm{r}=0.131, \mathrm{P}=0.23)$, positively correlated with LVDs $(\mathrm{r}=0.279, \mathrm{P}=0.009)$, IVS $(\mathrm{r}=0.360, \mathrm{P}=0.001)$, LVPW $(\mathrm{r}=0.287, \mathrm{P}=0.007)$, and negative correlated with FS $(r=-0.384, \mathrm{P}=0.000,<0.001)$, LVEF 

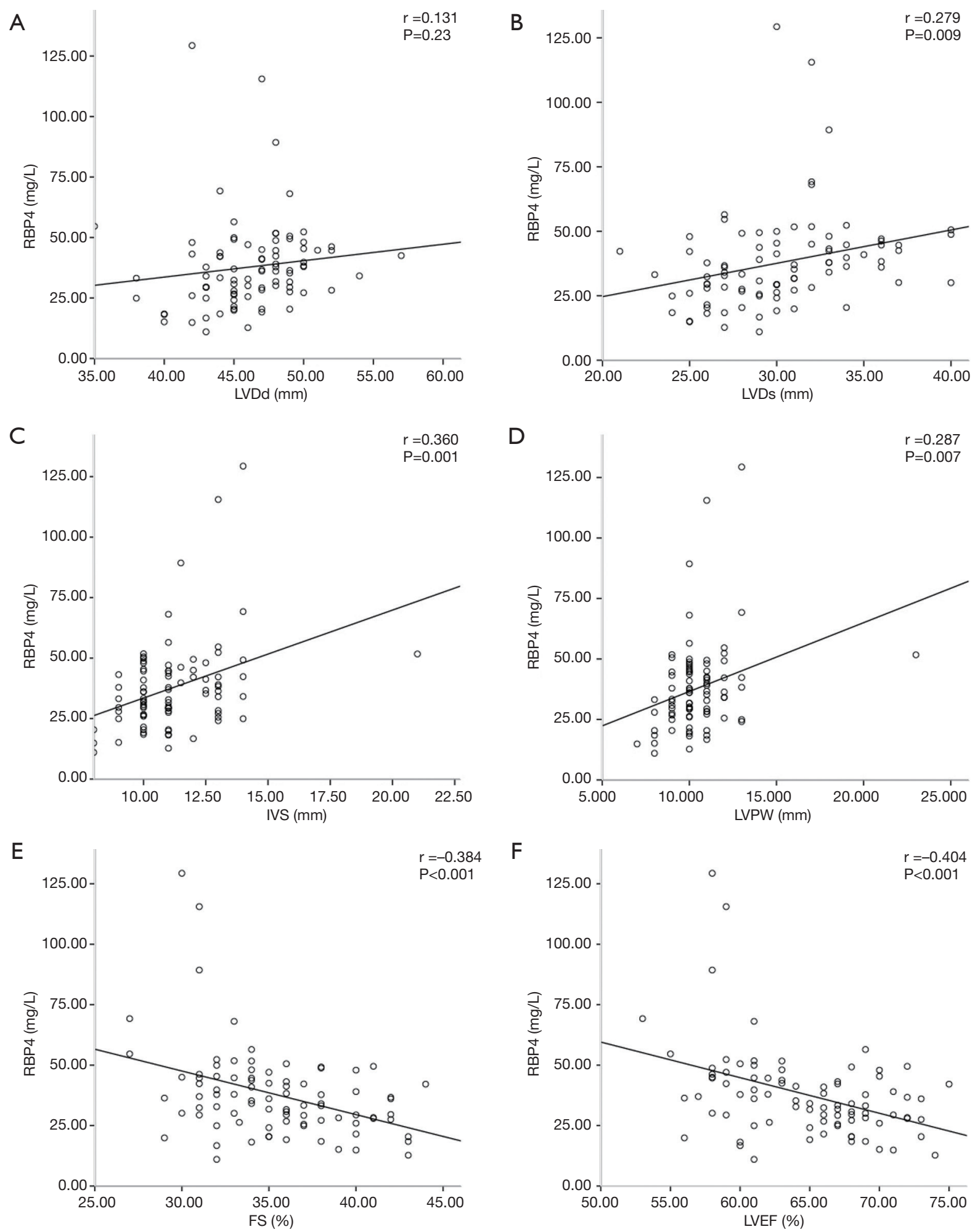

Figure 2 Curve estimation of relationship between plasma RBP4 level and LVDd (A), LVDs (B), IVS (C), LVPW (D), FS (E) and LVEF (F). RBP4, retinol binding protein 4; LVDd, left ventricular end-diastolic diameter; LVDs, left ventricular end-systolic diameter; IVS, interventricular septal thickness; LVPW, left ventricular posterior wall thickness; FS, fractional shortening; LVEF, left ventricular ejection fraction. 


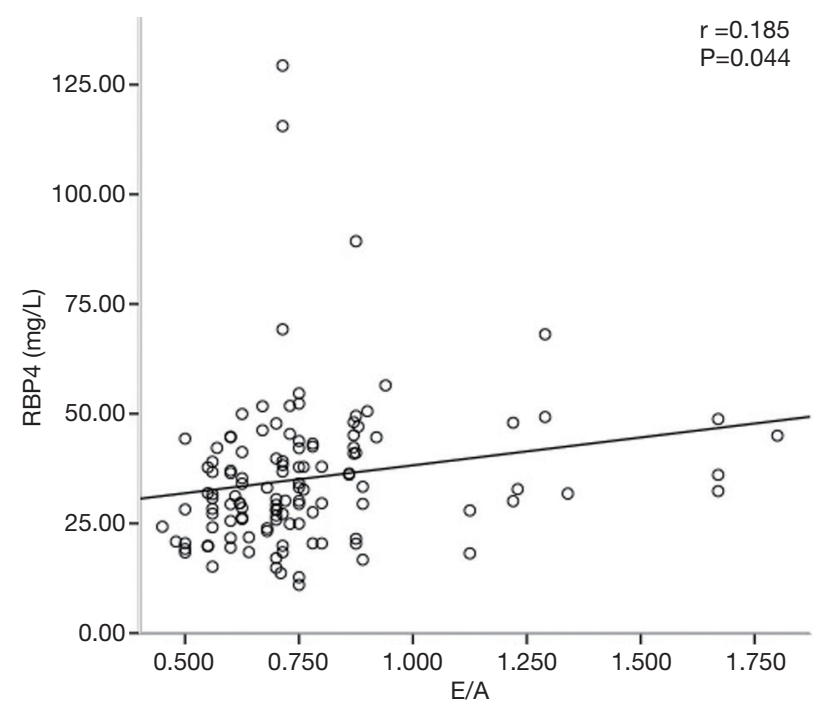

Figure 3 Pearson correlation analysis between plasma RBP4 level and E/A. Curve estimation of relationship between plasma RBP4 level and E/A: plasma RBP4 levels were correlated with the levels of $\mathrm{E} / \mathrm{A}(\mathrm{r}=0.185, \mathrm{P}<0.05)$. $\mathrm{E}$ : mitral valve early diastolic peak blood flow velocity; A: mitral valve late diastolic peak blood flow velocity. $\mathrm{RBP} 4$, retinol binding protein 4.

$(r=-0.404, P=0.000,<0.001)$. Finally, we further analyzed the correlation between RBP4 and left ventricular diastolic function. RBP4 was closely correlated with $\mathrm{E} / \mathrm{A}(\mathrm{r}=0.185$, $\mathrm{P}<0.05$ ) (Figure 3).

\section{Discussion}

The occurrence and development of hypertension are closely related to inflammatory response (12-14). Previous studies show that inflammatory reaction can promote proliferation, migration and differentiation of vascular cells, leading to damage of cardiac tissue stroma and collagen deposition, vascular dysfunction, myocardial hypertrophy, myocardial fibrosis and ventricular remodeling, causing deterioration of myocardial relaxation and myocardial compliance, and eventually resulting systolic and diastolic dysfunction (15). The mechanism underlying the pathogenesis of diastolic dysfunction is understudied. Therefore, it is very important to actively find an effective and specific cytokine as a specific target for diagnosis, treatment and progression of hypertension.

In this study, patients with $\mathrm{EH}$ who did not take medicine were taken as the research object. And, the relationship between serum RBP4 and the BP level and left ventricular diastolic function of patients with $\mathrm{EH}$ was determined. The results showed that RBP4 was closely related to the $\mathrm{BP}$ level of patients with $\mathrm{EH}$, and the A study involving 160 cases of prehypertension showed that (16) the RBP4 levels of prehypertension patients was significantly higher than that of normal BP patients $(\mathrm{P}<0.001)$, and $\mathrm{RBP} 4$ was still related to systolic and diastolic $\mathrm{BP}$ after adjusting for risk factors such as age, gender, smoking, drinking, etc. $(\mathrm{P}<0.001)$. A retrospective study confirmed that compared with the control group, the RBP4 levels in elderly male hypertensive patients were significantly increased (17). Animal experiments also confirmed that mice with RBP4 over-expression had increased BP levels $(\mathrm{P}<0.05)$, while the RBP4 knockout mice had decreased BP level $(\mathrm{P}<0.05)(18)$. Research showed that diastolic dysfunction was significantly related to RBP4 in severely obese subjects (19). These findings are consistent with our experimental results. Taken together, RBP4 is closely related to EH and our findings may provide new ideas for diagnosis and treatment of hypertension. However, the specific molecular mechanism that mediates the role of RBP4 in EH needs further study.

Early, comprehensive and accurate assessment of diastolic function in hypertensive patients is of great significance for improving their prognosis and preventing complications. The previous study reports that patients with $\mathrm{EH}$ will have changes in diastolic function at an early stage $(20,21)$. RBP4 is possibly involved in the occurrence and development of hypertension diastolic dysfunction and may act as an early predictor of left ventricular diastolic dysfunction. In summary, our findings highlight a significant correlation between RBP4 and diastolic function of $\mathrm{EH}$ and provide important pre-clinical data for the diagnosis and treatment of this disease.

\section{Acknowledgments}

Funding: This work was supported by grants from the National Natural Science Foundation of China (No. 81470501 and 81770440 to $\mathrm{X} \mathrm{Lu}$ ).

\section{Footnote}

Conflicts of Interest: The authors have no conflicts of interest to declare.

Ethical Statement: The authors are accountable for all aspects of the work in ensuring that questions related to the accuracy or integrity of any part of the work are 
appropriately investigated and resolved. This study was reviewed and approved by The Second Affiliated Hospital of Nanjing Medical University ethical review board (research number: 2015-KY-053), and all patients signed informed consent before enrollment.

\section{References}

1. NCD Risk Factor Collaboration (NCD-RisC). Worldwide trends in blood pressure from 1975 to 2015: a pooled analysis of 1479 population-based measurement studies with 19.1 million participants. Lancet 2017;389:37-55.

2. Go AS, Mozaffarian D, Roger VL, et al. Heart disease and stroke statistics--2013 update: A report from the American heart association. Circulation 2013;127:e6-245.

3. Tsao CW, Gona PN, Salton CJ, et al. Left ventricular structure and risk of cardiovascular events: a Framingham Heart Study Cardiac Magnetic Resonance Study. J Am Heart Assoc 2015;4:e002188.

4. Hu S, Ma S, Li X, et al. Relationships of SLC2A4, RBP4, PCK1, and PI3K Gene Polymorphisms with Gestational Diabetes Mellitus in a Chinese Population. Biomed Res Int 2019;2019:7398063.

5. Li JY, Chen XX, Lu XH, et al. Elevated RBP4 plasma levels were associated with diabetic retinopathy in type 2 diabetes. Biosci Rep 2018;38:BSR20181100.

6. Korek E, Gibas-Dorna M, Chęcińska-Maciejewska Z, et al. Serum RBP4 positively correlates with triglyceride level but not with BMI, fat mass and insulin resistance in healthy obese and non-obese individuals. Biomarkers 2018;23:683-8.

7. Bakshi S, Schmidt HM, Baskin AE, et al. Sexual dimorphism in developmental and diet-dependent circulating retinol binding protein 4 . Obes Sci Pract 2018;4:526-34.

8. Du C, Kong F. A Prospective Study of Maternal Plasma Concentrations of Retinol-Binding Protein 4 and Risk of Gestational Diabetes Mellitus. Ann Nutr Metab 2019;74:1-8.

9. Liu G, Sun Q, Chiuve SE, et al. Plasma Levels of Fatty Acid-Binding Protein 4, Retinol-Binding Protein 4, HighMolecular-Weight Adiponectin, and Cardiovascular Mortality Among Men With Type 2 Diabetes: A 22Year Prospective Study. Arterioscler Thromb Vasc Biol 2016;36:2259-67.

10. Chavarria N, Kate TS, Khan R, et al. Increased levels of retinol binding protein 4 in patients with advanced heart failure correct after hemodynamic improvement through ventricular assist device placement. Circ J 2012;76:2148-52.

11. Marschner RA, Pinto G, Borges J, et al. Short-Term Detraining does not Change Insulin Sensitivity and RBP4 in Rodents Previously Submitted to Aerobic Exercise. Horm Metab Res 2017;49:58-63.

12. Ryan MJ. An update on immune system activation in the pathogenesis of hypertension. Hypertension 2013;62:226-30.

13. Yao W, Sun Y, Wang X, et al. Elevated Serum Level of Interleukin 17 in a Population With Prehypertension. J Clin Hypertens (Greenwich) 2015;17:770-4.

14. Wang Q, Wang H, Wang J, et al. Angiotensin II-induced Hypertension is Reduced by Deficiency of P-selectin Glycoprotein Ligand-1. Sci Rep 2018;8:3223.

15. Agita A, Alsagaff MT. Inflammation, Immunity, and Hypertension. Acta Med Indones 2017;49:158-65.

16. Zhang JX, Zhu GP, Zhang BL, et al. Elevated serum retinol-binding protein 4 levels are correlated with blood pressure in prehypertensive Chinese. J Hum Hypertens 2017;31:611-5.

17. Majerczyk M, Choręza P, Bożentowicz-Wikarek M, et al. Increased plasma RBP4 concentration in older hypertensives is related to the decreased kidney function and the number of antihypertensive drugs-results from the PolSenior substudy. J Am Soc Hypertens 2017;11:71-80.

18. Kraus BJ, Sartoretto JL, Polak P, et al. Novel role for retinol-binding protein 4 in the regulation of blood pressure. FASEB J 2015;29:3133-40.

19. Porcar-Almela M, Codoñer-Franch P, Tuzón M, et al. Left ventricular diastolic function and cardiometabolic factors in obese normotensive children. Nutr Metab Cardiovasc Dis 2015;25:108-15.

20. Zhang X, Wu CE, Ye P, et al. Right ventricle may be involved in regional diastolic dysfunction earliest in primary hypertension patients. J Cell Biochem 2019;120:18088-93.

21. Chang HC, Cheng HM. P wave peak time: A time window to evaluate left ventricular diastolic function. J Clin Hypertens (Greenwich) 2019;21:616-7.

Cite this article as: $\mathrm{Li} \mathrm{X}$, Zhu S, Song G, Zhang K, Gao W, Huang J, Lu X. Retinol-binding protein 4 is closely correlated to blood pressure level and E/A in untreated essential hypertension patients. Ann Palliat Med 2019;8(5):645-650. doi: 10.21037/apm.2019.11.07 